\title{
Le REMI, réseau de surveillance de la qualité bactériologique des coquillages du littoral français: premiers résultats
}

\author{
Laurence Miossec \\ IFREMER - Laboratoire Surveillance du Milieu - Nantes Direction de l'Environnement et de l'A- \\ ménagement Littoral Rue de l'lle d'Yeu - BP 1049 - 44037 Nantes Cedex 01
}

Résumé. - Le REMI, réseau microbiologique, a un double objectif. II cherche à évaluer les niveaux et les tendances de la contamination bactériologique des eaux littorales françaises. II vise, également, à garantir la salubrité des coquillages destinés à la commercialisation.

Ce réseau est organisé en fonction de chacun de ces objectifs: le réseau de Surveillance correspond à l'objectif environnemental (niveaux et tendances), le réseau d'Intervention vise la protection du consommateur.

II a été réorganisé en 1989; les premiers résultats de son fonctionnement, sur des exemples choisis, sont présentés et discutés.

Mots-clés. - Surveillance - Coquillages - Bactériologie - Environnement - Santé publique.

\begin{abstract}
The microbiology network, REMI, has two objectives. To assess the bacteriological contamination levels and trends of the french marine environment and to ensure that shellfish are suitable for human consumption.

This network is organized according to each of these objectives: the Routine Survey is intented to accomplish the environmental objective (levels and trends), the Warning System ensures consumer protection.

It was restructured in 1989; the first results of its management on specific areas are investigated and discussed.
\end{abstract}

Key words. - Monitoring - Shellfish - Bacteriology - Environment - Public health.

\section{INTRODUCTION}

A l'interface des domaines continental et océanique, la frange littorale apparaît particulièrement vulnérable; elle reçoit, à la fois, les apports telluriques et anthropiques provenant des activités humaines (industrielles, urbaines et agricoles). La dégradation du milieu qui en résulte nuit au développement et au maintien de la pêche et de l'aquaculture. Une bonne gestion de la 
qualité du milieu passe par une connaissance exhaustive des apports et des niveaux de contamination en polluants de toutes sortes. Les programmes de surveillance établissent ces bilans et soulignent d'éventuels déséquilibres. De là peuvent être prises des mesures d'aménagement visant à supprimer ou réduire notablement les sources polluantes (Goldberg 1975, Wolfe et al. 1987).

Sur le littoral français, deux réseaux de surveillance existent, respectivement depuis 1974 et 1984 . Le premier, le RNO, réseau national d'observation, mesure les paramètres généraux de qualité (température, salinité, sels nutritifs, etc.) et, depuis 1979 , suit la contamination chimique le long du littoral (Claisse 1989). Le second, le REPHY, réseau phytoplanctonique, enregistre les perturbations phytoplanctoniques (Belin et al. 1989). Afin de compléter les connaissances qualitatives et quantitatives sur l'environnement marin, le REMI, réseau microbiologique, évalue, depuis 1989 , la contamination microbiologique des eaux littorales françaises. Nous présentons successivement les objectifs et les stratégies développés dans cette surveillance et quelques exemples de résultats obtenus.

\section{MISE EN PLACE DU REMI}

\section{Préambule}

De nombreux auteurs ont discuté les critères et les étapes nécessaires à la définition d'un programme optimum de surveillance de la pollution marine, citons Segar et Stamman (1986), Chapman et al. (1987). Statistiquement fiable, la Surveillance doit répondre à des besoins en terme d'aménagement et de gestion des ressources. A des objectifs clairement définis s'adaptent spécifiquement des stratégies intégrant les moyens disponibles. Paramètres et outils de surveillance doivent être sélectionnés par rapport aux questions posées.

L'appréciation de la contamination bactériologique du milieu marin et notamment des eaux conchylicoles est basée sur les indicateurs fécaux: coliformes et streptocoques. Sans grand danger sauf à de très fortes concentrations, ils ont été longtemps considérés comme indicateurs de germes pathogènes comme salmonelles et virus. L'amélioration des techniques analytiques de ces derniers a montré que les corrélations étaient, en fait, très variables; l'hypothèse de départ devait être réduite à la seule utilisation des germes fécaux comme traceurs de pollution (Bonde, 1977).

De nombreux programmes de recherche et de surveillance ont démontré l'utilité des bivalves comme organismes sentinelles capables d'indiquer les fluctuations du milieu et d'enregistrer les niveaux de pollutions marines, notamment chimiques (Goldbert et al. 1978). Les coquillages sont, en effet, sédentaires, largement répartis sur le littoral et leur mode d'alimentation par filtration permet l'accumulation de particules de différentes origines (algues unicellulaires, matière organique, bactéries et virus) (Wood 
1979). Leur utilisation comme intégrateurs de la qualité bactériologique des eaux douces et marines est très largement discutée dans la littérature (Trollope 1984, Prieur et al. 1990). Certains auteurs soulignent l'influence de facteurs extérieurs (température et salinité) et de paramètres physiologiques sur les capacités de filtration de l'animal et sur l'accumulation des germes conduisant à la comparaison délicate des résultats; ils émettent également des réserves sur l'aptitude des mollusques à enregistrer des phénomènes de très courte durée (Wood 1979, Delattre et Delesmont 1981). Par contre, d'autres scientifiques ont constaté que l'emploi de coquillages minimisait la variabilité observée dans l'eau et que ceux-ci constituaient, de ce fait, de bons intégrateurs de la pollution des eaux littorales. Ils permettent de localiser les sources de contamination des rejets d'eaux usées et des apports diffus et d'apprécier la conformité des zones conchylicoles en regard des normes sanitaires (Ayres et al. 1978, Turick et al. 1988, Plusquellec et al. 1990).

\section{Les stratégies}

Historiquement, l'objectif principal de la surveillance bactériologique des coquillages en France était la salubrité des bivalves pour la consommation humaine (Poggi 1986). Le REMI a été restructuré en 1989 dans un double but: surveiller l'environnement et garantir la salubrité des coquillages visà-vis du consommateur. La stratégie d'échantillonnage résultante répond à ces 2 objectifs.
Le littoral français a été initialement découpé en 92 secteurs: 56 zones conchylicoles et 36 zones présentant pour la plupart des potentialités aquacoles. La répartition de l'effort de surveillance par secteur a été effectuée proportionnellement à un indice intégrant production et salubrité locales et sur la base des capacités analytiques totales des laboratoires IFREMER impliqués dans la surveillance. Le nombre de stations retenues par secteur a été calculé en affectant la fréquence 1/12 (un prélèvement par mois) au nombre d'analyses (Miossec et Berthomé, sous presse).

Le réseau microbiologique est scindé en 2 parties: le réseau de Surveillance correspond à l'objectif environnemental, le réseau d'Intervention à l'objectif "Santé publique".

\section{Réseau de Surveillance}

Son but est d'évaluer les niveaux et les tendances de la contamination bactériologique du milieu marin mesurée au travers des coquillages pris comme intégrateurs. Les paramètres suivis sont les indicateurs classiques de pollution fécale, coliformes totaux et coliformes fécaux. Les résultats sont exprimés en nombre le plus probable (NPP) de coliformes dans $100 \mathrm{ml}$ de chair de coquillages et de liquide intervalvaire. La fréquence d'échantillonnage est mensuelle. Une couverture minimum de 2 stations de prélèvement a été affectée dans chacun des secteurs précédemment définis. De plus, l'effort de surveillance a été accentué dans les secteurs de production de coquillages. 
Actuellement, la couverture du littoral français est de 345 stations de prélèvement positionnées de façon systématique dans chaque secteur.

\section{Réseau d'Intervention}

L'objectif de ce réseau est de garantir la salubrité des coquillages sortant du milieu d'élevage. Parallèlement à l'analyse des coliformes totaux et fécaux, les salmonelles sont recherchées systématiquement. Seules les zones où il y a production et commercialisation de coquillages, sont concernées par cette surveillance. Le réseau d'Intervention est déclenché par des conditions météorologiques particulières (tempêtes, orages), certaines pratiques agricoles ou, plus exceptionnellement, par de mauvais résultats analytiques obtenus par le réseau de Surveillance ou d'autres sources de contrôle. La fréquence minimum d'Intervention est, alors, hebdomadaire. Le nombre de stations est de 278 tous secteurs confondus. Elles sont placées, à partir des sources de contaminations connues, suivant un gradient décroissant de pollution intégrant les phénomènes de dilution.

Ce schéma théorique de surveillance a été appliqué sur le littoral à partir d'avril 1989. En fonction de situations locales rencontrées, des adaptations ont été apportées principalement sur le nombre de points déterminés par le calcul de l'indice (fig. 1).

\section{RÉSULTATS}

Nous présentons, sur quelques exemples, les résultats du fonctionnement du réseau obtenus en Surveillance en 1989 et 1990 et en Intervention en 1989.

\section{Méthodes d'analyse}

Le dénombrement des coliformes fécaux se fait en milieu liquide en ensemençant trois séries de trois tubes avec l'échantillon et des dilutions décimales de celui-ci. L'interprétation numérique est donnée par la méthode du "Nombre le Plus Probable" (NPP) de coliformes fécaux dans 100 millilitres de coquillages et de liquide intervalvaire (broyat). Les coliformes totaux sont mis en évidence après une première série de cultures sur bouillon lactosé bilié au vert brillant à $37^{\circ} \mathrm{C}$ pendant 48 heures à l'étuve. Une deuxième série de cultures sur un milieu identique et sur eau peptonée à $44^{\circ} \mathrm{C}$ au bain-marie pendant 24 heures détermine les coliformes fécaux.

La recherche de salmonelles se fait sur 25 grammes de chair et de liquide intervalvaire et nécessite quatre phases successives : un préenrichissement en milieu non sélectif (eau peptonée tamponnée pendant 16 à 20 heures à $37^{\circ} \mathrm{C}$ ), un enrichissement en milieu sélectif (bouillon au sélénite à $37^{\circ} \mathrm{C}$ pendant 18 à 24 heures et simultanément Rappaport à $43^{\circ} \mathrm{C}$ pendant le même temps), isolement sur gélose au vert brillant et au rouge de phénol ainsi que sur Wilson-Blair pendant 20 à 24 heures à $37^{\circ} \mathrm{C}$, puis confirmation des colonies suspectes sur galerie API $20 \mathrm{E}$. Les résultats sont exprimés en terme de présence/absence. 


\section{Sites}

En surveillance, nous présentons les résultats obtenus en rivière d'Etel (Atlantique) et dans l'étang de Thau (Méditerranée) (fig. 1). Ce site est également présenté en Intervention. Prélèvements et analyses ont été réalisés par les équipes des laboratoires IFREMER respectivement de La Trinité/Mer et de Sète.

- Rivière d'Etel: 5 stations sont échantillonnées simultanément chaque mois. Les coquillages (Crassos- trea gigas) sont analysés le lendemain, suivant la technique décrite plus haut. Seuls les coliformes sont analysés.

- Etang de Thau: en Surveillance, les prélèvements sont réalisés sur 6 points mensuellement. En Intervention, 3 stations supplémentaires sont échantillonnées. Pendant la période de contamination les prélèvements ont été effectués 2 fois par semaine. Dans ce cas la teneur en coliformes et la présence de salmonelles ont été recherchées dans les coquillages (Crassostrea gigas).

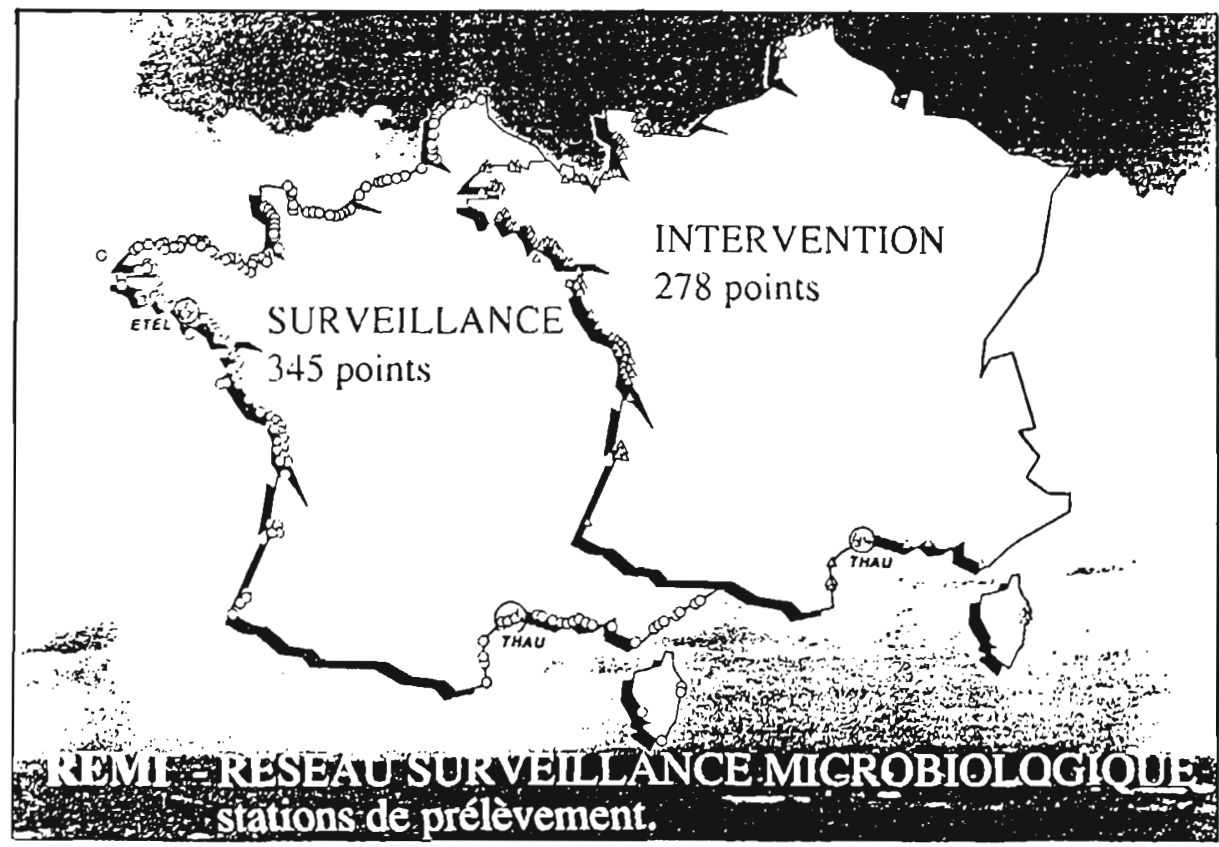

Fig. 1. - Surveillance et intervention sur le littoral fançais.

Fig. 1. - Routine Survey and Warning system along the french coast. 


\section{Résultats}

\section{Surveillance}

Les figures 2 et 3 présentent l'évolution moyenne mensuelle de la conta- mination bactérienne des coquillages, respectivement de la rivière d'Etel et de l'étang de Thau, entre avril 89 et décembre 90. (Les moyennes et les intervalles de confiance sont calculés après transformation logarithmique des

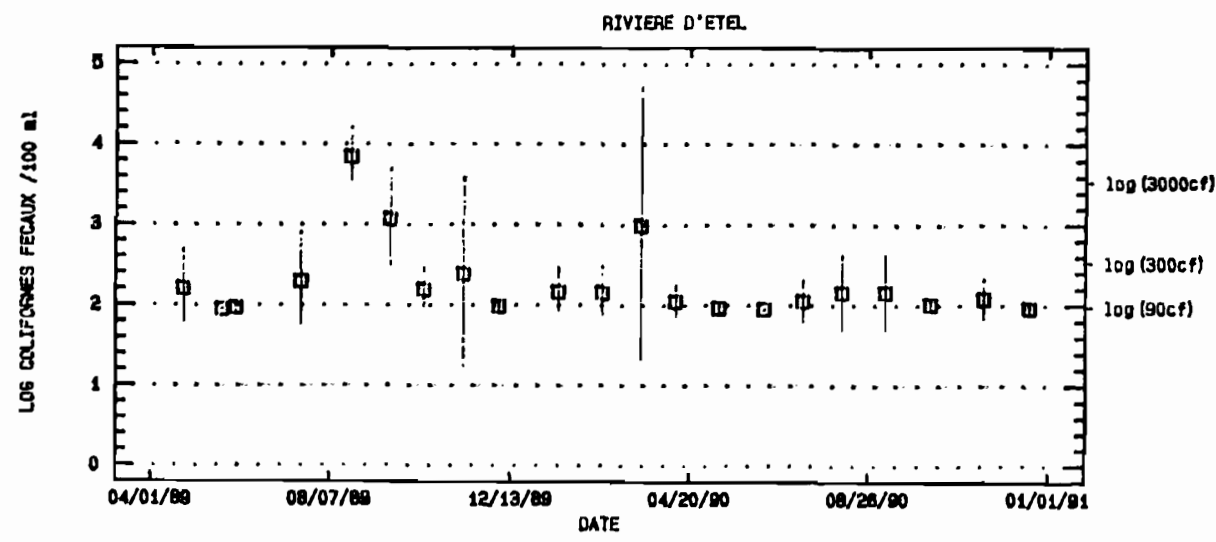

Fig. 2. - Contamination moyenne des coquillages de la rivière d'Etel (Surveillance).

Fig. 2. - Mean shellfish contamination in Etel River (Routine survey).

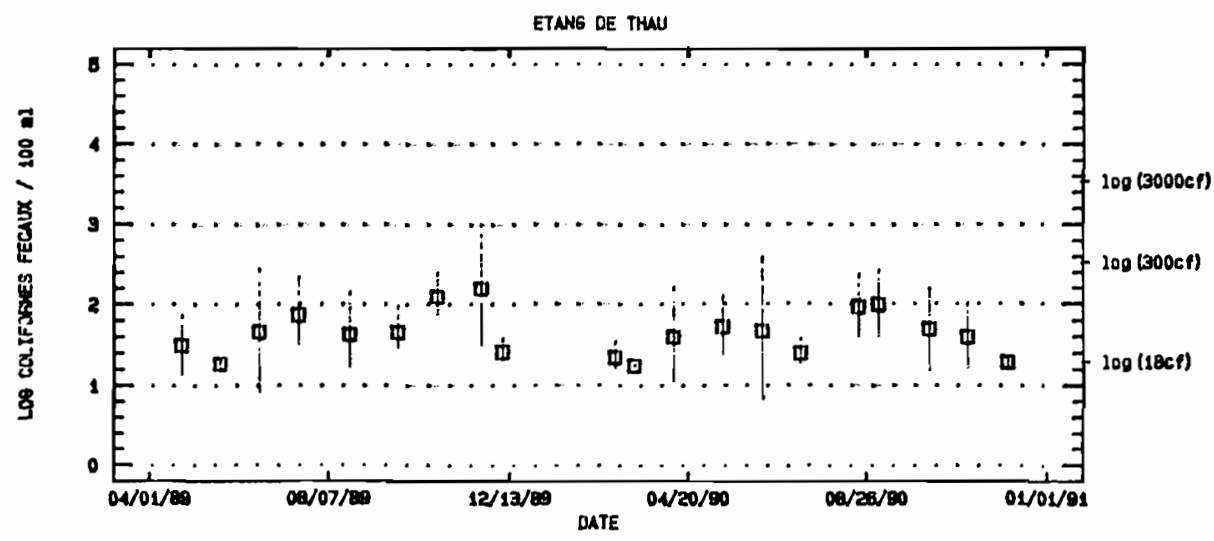

Fig. 3. - Contamination moyenne des coquillages de l'étang de Thau (Surveillance).

Fig. 3. - Mean shellfish contamination in Thau pond (Routine survey). 


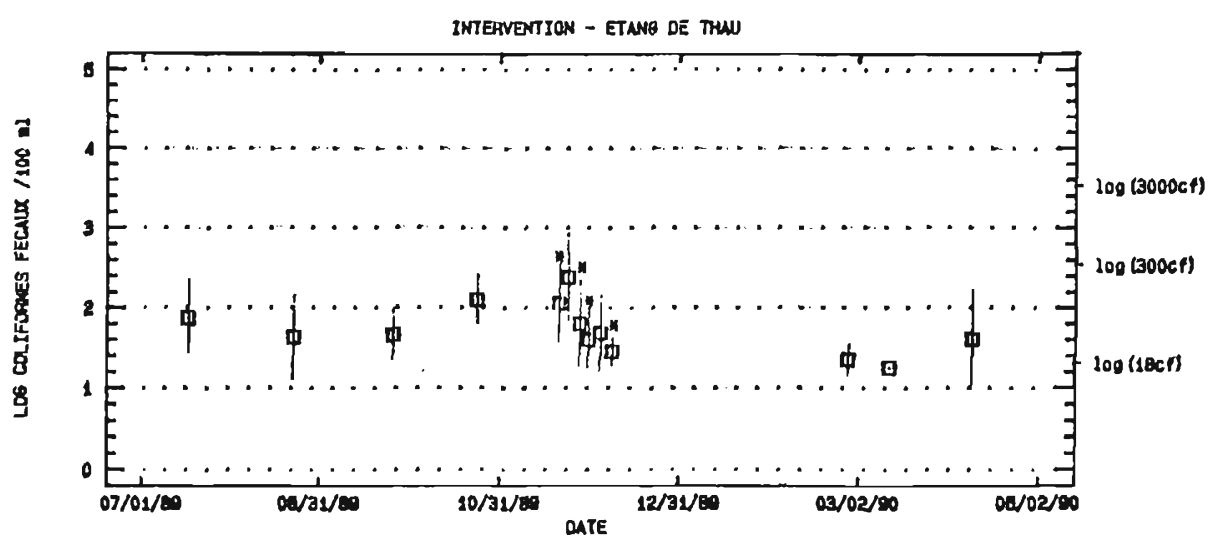

Fig. 4. - Concentrations moyennes des coquillages en coliformes fécaux pendant la période de contamination de l'étang de Thau (Intervention).

* Présence de salmonelles.

Fig. 4. - Mean shellfish concentrations in fecal coliforms during the contamination period in Thau pond (Warning system).

- Salmonella detection.

données. Les limites de détection sont de 1,95 (NPP $=90)$ pour le secteur d'Etel et de 1,25 (NPP = 18) pour Thau suivant la série de dilutions utilisées).

Dans l'un et l'autre secteurs, on remarque des périodes de contamination importante, de plus grande amplitude toutefois en rivière d'Etel. L'intervalle de confiance permet d'apprécier la dispersion autour de la moyenne. On peut constater ainsi que la contamination enregistrée en août 89 était générale sur toute la rivière d'Etel; elle était plus localisée en mars 90 (sur deux points du centre de la rivière). La contamination est plus homogène sur l'ensemble de l'étang de Thau.

\section{Intervention}

De fortes précipitations sur l'étang de Thau et son bassin versant pendant la période du 16 au 22 novembre 1989 ont provoqué le déclenchement du réseau bactériologique "Intervention" le 20 novembre (Angeli com. pers.). Le tableau I et la figure 4 présentent les résultats obtenus entre juillet 89 et mai 90 ; les moyennes obtenues en novembre et décembre 89 ont été calculées à partir des résultats d'intervention, celles des autres mois à partir des résultats de surveillance.

La contamination fécale est apparue marquée au début de la période d'intervention. Elle était, cependant, hétérogène sur l'ensemble du bassin. Elle a, rapidement, diminué pour atteindre, dès le 20 novembre, des valeurs moyennes comparables à la normale. Au cours de cette période des maxima de 1440 coliformes fécaux dans $100 \mathrm{ml}$ de chair et de li- 
Tableau I. - Contamination bactérienne de l'étang de Thau pendant la période d'Intervention.

() nombre de stations avec salmonelles.

Table I. - Bacterial contamination in Thau pond during the warning system period. () number of sampling stations with salmonella.

\begin{tabular}{|c|c|c|c|c|c|}
\hline & Date & $\begin{array}{l}\text { Nombre de } \\
\text { prélèvements }\end{array}$ & $\begin{array}{l}\text { Concentration moyenne } \\
\text { en coliformes fécaux } \\
/ 100 \mathrm{ml} \text { de broyat }\end{array}$ & $\begin{array}{l}\text { Intervalle de } \\
\text { confiance } \\
\text { (à } 5 \% \text { ) }\end{array}$ & $\begin{array}{l}\text { Présence de } \\
\text { salmonelle }\end{array}$ \\
\hline 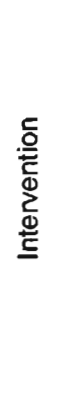 & $\begin{array}{l}17 / 07 / 89 \\
22 / 08 / 89 \\
25 / 09 / 89 \\
23 / 10 / 89 \\
20 / 11 / 89 \\
23 / 11 / 89 \\
27 / 11 / 89 \\
30 / 11 / 89 \\
04 / 12 / 89 \\
08 / 12 / 89 \\
26 / 02 / 90 \\
12 / 03 / 90 \\
09 / 04 / 90\end{array}$ & $\begin{array}{l}6 \\
6 \\
6 \\
6 \\
9 \\
9 \\
9 \\
9 \\
9 \\
9 \\
6 \\
6 \\
6\end{array}$ & $\begin{array}{l}1,874 \\
1,637 \\
1,666 \\
2,097 \\
2,054 \\
2,386 \\
1,802 \\
1,603 \\
1,687 \\
1,456 \\
1,357 \\
1,255 \\
1,606\end{array}$ & $\begin{array}{l}0,479 \\
0,530 \\
0,329 \\
0,317 \\
0,498 \\
0,558 \\
0,553 \\
0,362 \\
0,487 \\
0,234 \\
0,194 \\
0,000 \\
0,619\end{array}$ & $\begin{array}{l}- \\
- \\
- \\
- \\
+(1) \\
- \\
+(2) \\
+(2) \\
- \\
+(1) \\
- \\
- \\
-\end{array}$ \\
\hline
\end{tabular}

quide intervalvaire (NPP) ont été enregistrés sur plusieurs stations.

Les analyses ont montré la présence de salmonelles dès le premier échantillonnage. Une à deux stations se sont révélées positives chaque semaine sur l'ensemble de l'étang de Thau (tableau I).

\section{DISCUSSION - CONCLUSIONS}

Les normes françaises et européennes concernant la qualité des eaux conchylicoles sont basées sur la contamination bactériologique des coquillages exprimée par le nombre le plus probable de coliformes fécaux trouvés dans 100 millilitres de chair de coquillages broyée et diluée. La valeur guide est de 300 coliformes fécaux/100 ml. Suivant les textes, un pourcentage de dépassement est toléré; le texte français n'accepte, cependant, pas de valeur supérieure à 3000 coliformes fécaux.

En référence à ces normes, nous pouvons constater que l'étang de Thau présente des résultats conformes à la notion de salubrité pendant la période étudiée. Par contre, les résultats obtenus en rivière d'Etel soulignent des phénomènes périodiques de contamination pouvant être intenses si on en juge par la valeur moyenne obtenue en août 89 .

Cette première analyse des résultats de surveillance reste sommaire, la période d'étude est encore trop limitée. Cependant, au travers de ces 2 exemples, nous avons voulu mon- 
trer qu'il était possible, sur la base des résultats de surveillance, d'apprécier la qualité d'une zone de production conchylicole. L'observation de contamination locale ponctuelle ou chronique doit conduire à des analyses plus fines visant à rechercher les causes de ces pollutions: apports par les rivières, par les eaux de ruissellement, absence de réseau d'assainissement, mauvais fonctionnement d'une station d'épuration, etc. Des mesures d'aménagement doivent découler des conclusions apportées.

Si les eaux conchylicoles de l'étang de Thau sont salubres la majeure partie de l'année, on enregistre des augmentations de la colimétrie des eaux et des coquillages à la suite de fortes pluies (Lebaron et al. 1990). Ces contaminations ont plusieurs origines. Certaines communes ont des réseaux d'assainissement de type unitaire. Après de fortes pluies, le mélange des eaux pluviales et des eaux usées est évacué sans traitement par des déversoirs d'orage, directement dans l'étang, entrainant une contamination microbienne. De même, les eaux de ruissellement constituent une source de pollution non négligeable par lessivage du bassin versant à dominance agricole (viticulture). En novembre 1989, il y a eu convergence de deux phénomènes météorologiques: de fortes pluies faisant suite à une période de sécheresse et la persistance de vent marin limitant le renouvellement des eaux de l'étang (Angeli, com. pers.). Le déclenchement du réseau d'Intervention a permis d'enregistrer la contamination bactérienne résultante dans les coquillages. La teneur en coliformes fécaux a rapidement diminué avec le temps, cependant les salmonelles ont persisté. II apparaît, en effet, une survie différentielle entre ces germes bactériens en fonction des paramètres physiques du milieu. A basse température, les salmonelles peuvent survivre plus longtemps qu'Escherichia coli (Rhodes et Kator, 1988).

L'objectif d'un contrôle sanitaire est de vérifier que le produit est dépourvu de substances toxiques et de germes pathogènes (Wood 1972). La réglementation française en la matière impose une absence de salmonelle dans les coquillages bivalves présentés vivants à la vente. Cette règle et la représentativité relative des coliformes vis-à-vis du risque sanitaire nous ont amené à analyser conjointement coliformes et salmonelles en Intervention. Le déclenchement de celui-ci conduit à l'établissement de relations étroites avec les services administratifs chargés d'appliquer les textes. Lorsque les teneurs en germes bactériens dans les coquillages atteignent des valeurs susceptibles de nuire à la santé humaine, ces services sont informés des résultats et prennent des mesures officielles d'interdiction de commercialisation des coquillages provenant des secteurs incriminés. L'interdiction est levée lorsque les résultats sont revenus à des niveaux acceptables. Dans le cas de l'étang de Thau, la contamination enregistrée a conduit à une période de fermeture de 45 jours au total. La présence de salmonelle a été obser- 
vée pendant toute cette période ; précisons que des analyses, menées régulièrement auparavant, concluaient à l'absence de salmonelle.

Nous avons voulu dans la partie Intervention du REMI définir des règles de gestion des bassins conchylicoles en terme de salubrité des coquillages vis-à-vis des consommateurs sur la base d'une réglementation en vigueur et en fonction des connaissances disponibles. Les mesures d'interdiction de commercialisation prises par les services administratifs peuvent être accompagnées de dérogation lorsque les conchyliculteurs disposent de moyens à terre (bassins oxygénés insubmersibles) permettant l'épuration des coquillages. Une stratégie de surveillance ne doit pas être figée. Elle doit être améliorée en fonction des résultats obtenus par le programme de surveillance lui-même et par les apports de la recherche (Segar et Stamman 1986). Parallèlement au fonctionnement du REMI, des études sont en cours pour juger de la représentativité de l'échantillonnage et pour mesurer les sources de variabilité liée à la méthodologie et aux fluctuations spatio-temporelles. De même, l'amélioration des techniques analytiques doit conduire dans le domaine des germes indicateurs à obtenir des réponses plus rapides, utiles lors des phénomènes de contamination impliquant des interdictions de commercialisation. Les recherches sur le devenir des germes d'origine entérique en zone littorale permettent la compréhension des conditions de survie des indicateurs de contamination et des germes pathogènes en milieu marin. Un effort important doit également être mené dans le domaine de l'épidémiologie afin de mieux apprécier les risques vis-à-vis du consommateur.

\section{BIBLIOGRAPHIE}

Ayres P.A., Burton H.W., Cullum M.L., 1978. Sewage pollution and shellfish in Techniques for the study of mixed populations, Society for applied bacteriology. Technical series 11 Ac. Press: 51-62.

Belin C., Berthomé J.P., Lassus P., 1989. Dinoflagellés toxiques et phénomènes d'eau colorées sur les côtes françaises: évolution et tendances entre 1975 et 1988. Hydroécol. appl. 1/2: 3-17.

Bonde G.J., 1977. Bacterial indication of coater pollution. Adv. Aquati. microbiol 1: 273-364.

Chapman P.M., Dexter R.N., Goldstein L., 1987. Development of monitoring programmes to assess the long-term health of aquatic ecosystems. A model from puget Sound USA. Mar. Pollut. bull, vol. 18, $\mathrm{n}^{\circ} 10$ : 521-527.

Claisse D., 1989. Chemical contamination of french coasts, the results of a ten years mussel watch. Mar. Pollut. bull. vol. 20, n $n^{\circ}$ 10: 523-528.

Delattre J.H. et Delesmont R., 1981. L'analyse de coquillages peut-elle servir au contrôle microbiologique du littoral? Rev. Int. Oceanogr. Med. Tomes LXII-LXIV: 11-16.

Goldberg E.D. 1975. The mussel watch, a first step in global marine monitoring. Mar. Pollut. bull., volume $6, \mathrm{n}^{\circ} 7$, p. 111.

Goldberg E.D., Bowen V.T., Farrington J.W., Harvey G., Martin J.H., Parker P.L., Risebrough R.W., Robertson W., Schneider E., Gamble E., 1978. The 
Mussel Watch. Environmental Conservation, vol. $5, \mathrm{n}^{\circ}$ 2: 101-125.

Lebaron P., Trousselier M., Got P., Baleux B., 1990. Distribution spatio-temporelle d'une population bactérienne allochtone (coliformes thermotolérants) dans un écosystème marin côtier (Bassin de Thau, France) Can. J. Microbiol. Vol. 36: 307-317.

Miossec L., Berthomé J.P. (sous presse). Le réseau de surveillance microbiologique du littoral français (REMI). Actes du colloque UOF "Le littoral, ses contraintes environnementales et ses conflits d'utilisation " Nantes 1-4 juillet 1991. 5 pages.

Plusquellec A., Beucher M., Prieur D., Le Gal Y., 1990. Contamination of the mussel, Mytilus edulis linnaeus, 1758, by enteric bacteria. Journ. of Shellfish Res. Vol. 9, n 1: 95-101.

Poggi R., 1986. Qualités requises des eaux conchylicoles et organisation de leur surveillance. TSM, L'eau $n^{\circ} 11$ : 530-538.

Prieur D., Mével G., Nicolas J.L., Plusquellec A., Vigneulle H., 1990. Interactions between bivalve molluscs and bacteria in the marine environment. Oceanogr. Mar. biol. Ann. Rev., 28: 277-352.

Rhodes M.W. et Kator H., 1988. Survival of Escherichia coli and Salmonella spp. in estuarine environments, Applied and environmental microbiology, vol. 54, no 12, 2902-2507.

Segar D.A., Stamman E, 1986. Fundamentals of marine pollution monitoring programme design. Mar. Pollut. bull., vol. 17, $n^{\circ}$ 5: 194-200.

Trollope D.R., 1984. Use of molluscs to monitor bacteria in water in Microbiological methods for environmental biotechnology ed. by J.M. Grainger and J.M. Lynch, Ac. Press: 393-408.

Turick C.E., Sexstone A.J., Bissonette G.K., 1988. Freshwater mussels as monitors of bacteriological water quality. Water, Air and Soil pollution, 40 : 449-460.

Wolfe D.A., Champ M.A., Flemer D.A., Mearns A.J., 1987. Long-term biological data sets: their role in research, monitoring and management of estuarine and coastal marine systems. Estuaries, vol. 10, $\mathrm{n}^{\circ}$ 3: 181-193.

Wood P.C., 1979. Public health aspects of shellfish from polluted waters in Biological indicators of water quality ed. by A. James and L. Evison: 13-1, 13-15.

Wood P.C., 1972. The principles and methods employed for the sanitary control of molluscan shellfish. Marine poll. and sea life, Ruivo M., West byfleet and London fishing news. 